\title{
Effets de la loi sur le travail sur la formation postgraduée des médecins et temps consacré à cette dernière: enquête auprès des responsables d'établissements de formation
}

Au printemps 2006, les responsables de 1370 établissements de formation reconnus ont été invités à répondre à un questionnaire portant sur les trois sujets suivants: résultats du sondage effectué en 2005 auprès des médecinsassistants, effets de la loi sur le travail sur la formation postgraduée et sur la prise en charge des patients, temps consacré à la formation postgraduée par les intéressés. Alors que les médecins-assistants estiment que ni la formation postgraduée ni la prise en charge ne sont affectées par la loi précitée, les responsables décèlent en revanche un effet négatif de celle-ci dans ces deux domaines. La moitié des responsables d'établissement consacre par ailleurs plus de six heures par semaine à la formation postgraduée des médecins. Ce chiffre reflète l'importance que les responsables attachent à la formation, mais l'estimation des heures qui lui sont consacrées ne permet pas de tirer des conséquences quant à son coût ou à son efficience.

Michael Siegrist ${ }^{a}$,

Pascale Orlow ${ }^{a}$, Max Giger ${ }^{b}$

a Institut de recherches sociales de l’Université de Zurich

b membre du comité central de la FMH, domaine Formation médicale
Correspondance

Dr Max Giger

FMH

Elfenstrasse 18

CH-3000 Berne 15

max.giger@hin.ch
Pour pouvoir décrire fiablement la situation de la formation postgraduée, il convient d'interroger régulièrement non seulement les médecins-assistants [1], mais aussi les responsables des établissements de formation. L'enquête réalisée auprès de ces derniers en 2004 a montré que des mesures d'une ampleur réjouissante avaient été prises pour améliorer la qualité de la formation postgraduée suite aux résultats du sondage effectué chez les assistants [2]. Cette enquête a aussi révélé qu'une majorité de responsables considère que les médecins-assistants montrent moins d'ardeur à la tâche qu'auparavant.

Notre but en lançant l'enquête 2006 auprès des responsables d'établissements était de voir si ceux-ci continuent dans l'ensemble à juger positivement le sondage des assistants et à prendre des mesures d'amélioration de la qualité en fonction de ses résultats. Il est ressorti de la version 2005 dudit sondage qu'une majorité des médecins-assistants considère que la loi sur le travail n'a pas d'effets négatifs sur leur formation postgraduée et leur activité professionnelle [1]. Dans la présente analyse, nous avons examiné la question de savoir comment les responsables éva- luent l'influence qu'exerce la loi sur le travail sur la formation et le travail des assistants et ce, en comparaison avec l'appréciation fournie par les assistants eux-mêmes. Finalement, nous avons établi un relevé du nombre d'heures que les responsables consacrent en moyenne par mois à la formation des médecins-assistants.

\section{Méthode}

\section{Echantillon}

L'enquête statistique effectuée auprès des responsables d'établissements de formation comportait des questions touchant à des aspects divers de la formation postgraduée. Elle a été adressée au printemps 2006 par voie électronique à 1370 établissements de formation postgraduée reconnus par la FMH. La question ayant obtenu le taux de retour le plus bas a reçu une réponse de la part de $62 \%$ des responsables. Ceux-ci étaient tout d'abord invités à indiquer leur fonction au sein de l'établissement. 80,3\% des participants ont répondu par «médecin-chef», 14,5\% par «médecin adjoint», $0,9 \%$ par «chef de clinique» et $4,3 \%$ par «autre». Les résultats de l'en- 
quête relatifs à la loi sur le travail ont été comparés à ceux du sondage réalisé auprès des médecins-assistants à la fin de l'été 2005.

\section{Questionnaire}

Les personnes sondées devaient avant tout prendre position sur un certain nombre d'affirmations. Six réponses étaient possibles, allant de 1 «c'est totalement inexact» à 6 «c'est parfaitement exact». Les chiffres intermédiaires n'étaient pas décrits verbalement. Outre le relevé annuel de la structure des établissements, le questionnaire s'articulait en trois domaines thématiques: des questions étaient tout d'abord posées sur le rapport concernant la qualité de la formation en

Figure 1

Formation postgraduée: réponses concernant l'affirmation «La réglementation du temps de travail régie par la loi a des effets négatifs sur la formation postgraduée des médecins-assistants».

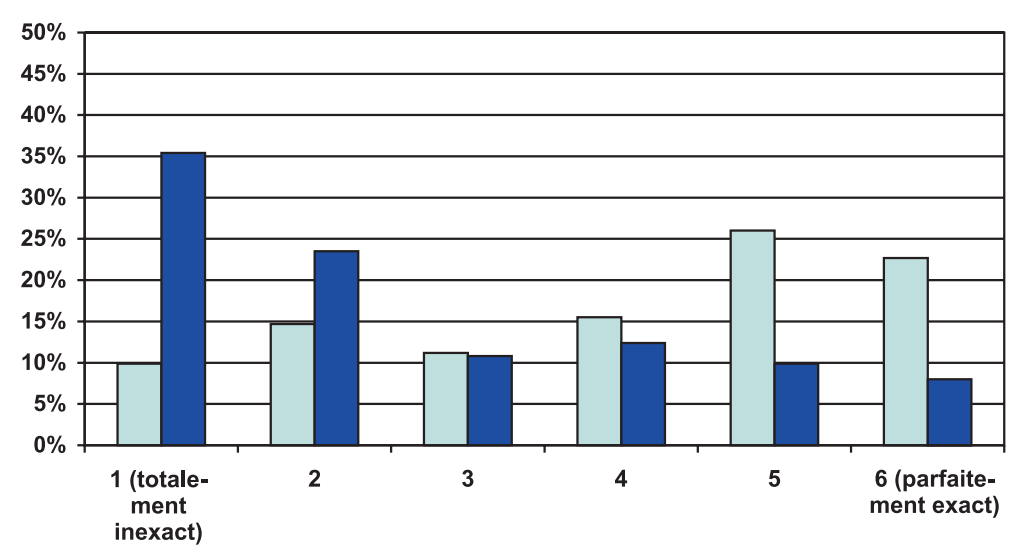

$\square$ Responsables EFP ( $\mathbf{N = 1 0 3 2 )} \square$ Médecins-assistants ( $\mathbf{N = 5 3 0 9 )}$

Figure 2

Soins aux patients: réponses concernant l'affirmation «La réglementation du temps de travail régie par la loi a des effets négatifs sur les soins donnés aux patients par les médecins-assistants».

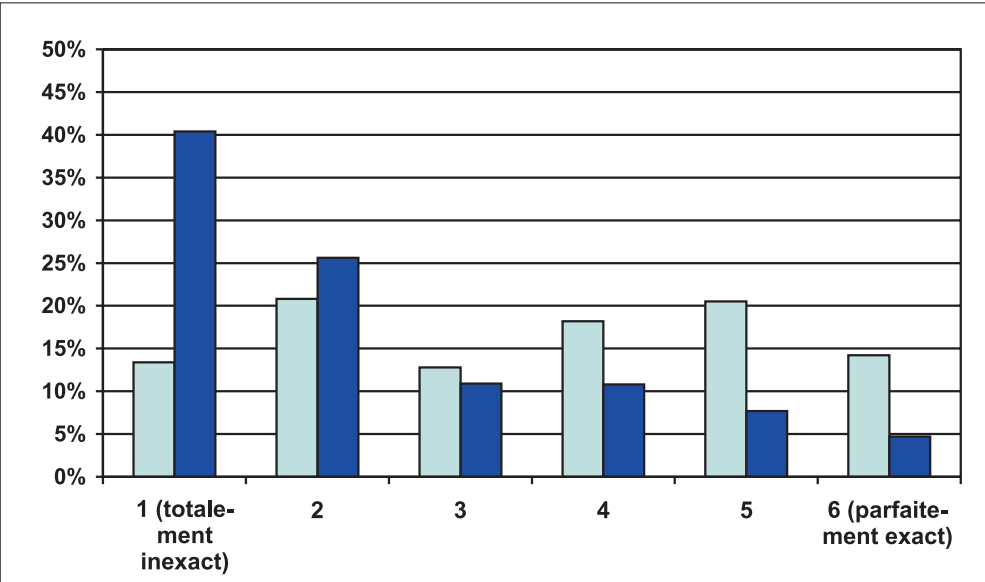

$\square$ Responsables EFP ( $N=1020) \square$ Médecins-assistants ( $N=5304)$

2005 vue par les médecins-assistants, puis sur la situation relative à la formation postgraduée et à l'activité professionnelle des assistants, et enfin sur le temps consacré par les responsables à la formation des assistants. Les responsables de 1000 établissements ayant reçu un exemplaire du rapport se voyaient en outre poser des questions spécifiques à son sujet. Ils pouvaient mentionner les activités que ce rapport les avaient incités à entreprendre. Le libellé précis des questions figure en annexe au présent article.

Les responsables étaient invités à indiquer combien de temps ils consacrent en moyenne par mois à diverses activités s'inscrivant dans la formation postgraduée des médecins-assistants. Les six secteurs d'activité suivants étaient nommés: entretiens de feedback concernant la formation postgraduée des médecins-assistants, réunions de cadres pour l'évaluation des médecins-assistants, préparation personnelle pour la formation postgraduée des médecins-assistants, exposés pour médecins-assistants, instructions et directives au chevet du patient (Bedsideteaching/assistanat en chirurgie).

\section{Résultats}

Appréciation du rapport concernant la qualité de la formation en 2005 vue par les médecins-assistants Dans $77 \%$ * $(\mathrm{N}=720)$ des établissements, les résultats ont été discutés avec les chefs de clinique/médecins adjoints et dans $67 \%(\mathrm{~N}=620)$ des établissements, avec les médecins-assistants. Une action concrète a été entreprise pour améliorer la qualité de la formation dans $60 \%$ $(\mathrm{N}=554)$ des établissements. Enfin, les résultats du sondage ont incité $65 \%(\mathrm{~N}=554)$ des responsables d'établissements de formation à repenser la formation postgraduée.

\section{Loi sur le travail}

La figure 1 présente les réponses à la question de savoir si la réglementation légale du temps de travail a des effets négatifs sur la formation postgraduée des médecins-assistants. Cette figure montre que les responsables d'établissements évaluent la situation de façon nettement différente que les assistants: alors que plus de 50\% des responsables sont d'avis que la réduction du temps de travail se répercute négativement sur la formation postgraduée, une majorité des médecins-assistants pensent que ce n'est pas le cas.

Les réponses concernant les effets de la réglementation du temps de travail sur les soins donnés aux patients par les médecins-assistants débouchent sur un tableau semblable (fig. 2). La 
majorité des assistants ne constate aucune influence négative. Chez les responsables d'établissements en revanche, l'avis prédomine selon lequel un effet négatif se fait sentir sur l'activité des assistants au chevet du patient.

\section{Temps consacré à la formation postgraduée} par les responsables d'établissements

Une analyse exploratoire des données a révélé l'existence de cas extrêmes («outliners» ou valeurs extrêmement élevées). C'est pourquoi seuls des chiffres indicatifs solides comme la médiane et l'intervalle interquartile (IIQ) sont présentés ciaprès, deux valeurs qui ne sont pratiquement pas influencées par les cas extrêmes. Les heures indiquées en regard des six aspects de la formation postgraduée considérés ont été additionnées. La médiane pour l'ensemble des disciplines $(\mathrm{N}=806)$ se monte à 24 heures par mois (IIQ $=24,3$ ) .

Les chiffres du tableau 1 représentent le temps que les responsables d'établissements des sept disciplines comptant le plus de médecins-assistants estiment consacrer à la formation postgraduée. La médiane des diverses disciplines oscille entre 20 et 32 heures par mois.

\section{Discussion}

Une grande partie des responsables déclare avoir remis en question la formation postgraduée dispensée par son établissement, voire induit des processus de changement dans ce domaine, sur la base des résultats du sondage effectué auprès des médecins-assistants. Il s'agit là d'un résultat fort réjouissant, qui montre notamment que ce sondage a atteint l'objectif consistant à donner aux responsables d'établissements des indications aussi concrètes que possibles sur les potentiels de changement en matière de formation.

Quant aux effets de la loi sur le travail, assistants et responsables les évaluent de manière très différente. La plupart des médecins-assistants

\section{Tableau 1}

Médiane et IIQ du temps que les responsables d'établissements consacrent mensuellement à la formation selon leur propre estimation; liste établie en fonction des sept disciplines comptant le plus de médecins-assistants.

\begin{tabular}{|c|c|c|c|}
\hline Discipline médicale & Médiane (heure/mois) & IIQ & $\mathrm{N}$ \\
\hline Anesthésiologie & 32,0 & 35,9 & 34 \\
\hline $\begin{array}{l}\text { Chirurgie orthopédique et traumatologie } \\
\text { de l'appareil locomoteur }\end{array}$ & 28,1 & 30,0 & 33 \\
\hline Chirurgie & 27,5 & 23,2 & 75 \\
\hline Pédiatrie & 27,0 & 23,6 & 27 \\
\hline Médecine interne & 27,0 & 29,5 & 125 \\
\hline Gynécologie et obstétrique & 22,3 & 23,6 & 50 \\
\hline Psychiatrie et psychothérapie & 20,0 & 18,6 & 71 \\
\hline
\end{tabular}

estiment que cette loi n'a pas de répercussions négatives sur leur formation ou leur activité au chevet du patient. Selon les responsables d'établissements, on constate en revanche des effets négatifs dans ces deux domaines. Une explication possible de cette différence d'appréciation pourrait résider dans le fait que les deux groupes disposent d'un champ d'expérience entièrement différent et utilisent par conséquent une autre échelle de comparaison.

L'enquête réalisée auprès des responsables montre par ailleurs que ceux-ci consacrent beaucoup de temps à la formation postgraduée des médecins. Cinquante pour cent des sondés déclarent s'en occuper plus de 24 heures par mois. La moitié des responsables d'établissements des sept disciplines comptant le plus de médecinsassistants lui dédient entre 5 et 8 heures par semaine (fig. 3). Ces chiffres se situent dans les limites des relevés effectués en 2000 et 2002 dans les hôpitaux universitaires de Lausanne et de $\mathrm{Zu}$ rich $[3,4]$.

En interprétant ces indications d'heures, il convient toutefois de tenir compte du fait qu'il s'agit d'une part d'appréciations rétrospectives et d'autre part d'auto-estimations. Il peut aussi arriver que des questions soient interprétées différemment par les personnes sondées. On ne pourrait supprimer ces inconvénients qu'en établissant un relevé très précis des activités des responsables, fondé sur une définition détaillée des activités attribuables à la formation. Une telle approche impliquerait un investissement financier important. On ajoutera à cela que les chiffres présentés ici ne représentent que l'investissement en temps des responsables d'établissements. Si l'on y ajoutait les heures consacrées à la formation des assistants par les médecins-adjoints, les chefs de clinique et le personnel paramédical, ces chiffres seraient plus élevés en conséquence. Pour pouvoir formuler des conclusions fiables au sujet de l'efficacité et de l'efficience de la formation postgraduée, il faudrait en outre comparer les chiffres concernés aux prestations fournies par les médecins-assistants.

\section{Références}

1 Siegrist M, Orlow P, Giger M. Weiterbildung aus der Sicht der Assistenzärzte: Die wichtigsten Resultate der Umfrage 2004 bei Assistenzärztinnen und -ärzten über die Weiterbildung. Schweiz Ärztezeitung 2005;86(7):412-23.

2 Siegrist M, Giger M. Assistenten- und Assistentinnenumfrage 2003 aus Sicht der Leiterinnen und Leiter von Weiterbildungsstätten. Schweiz Ärztezeitung 2004;85(42):2262-6.

3 Schenker L. Communication personnelle.

4 Vetter W. Communication personnelle. 
Figure 3

Heures que les responsables d'établissements consacrent par semaine à la formation postgraduée selon leur propre estimation; liste établie en fonction des sept disciplines comptant le plus de médecins-assistants. Les réponses mentionnant $20 \mathrm{~h} /$ semaine ou davantage ne sont pas prises en considération.

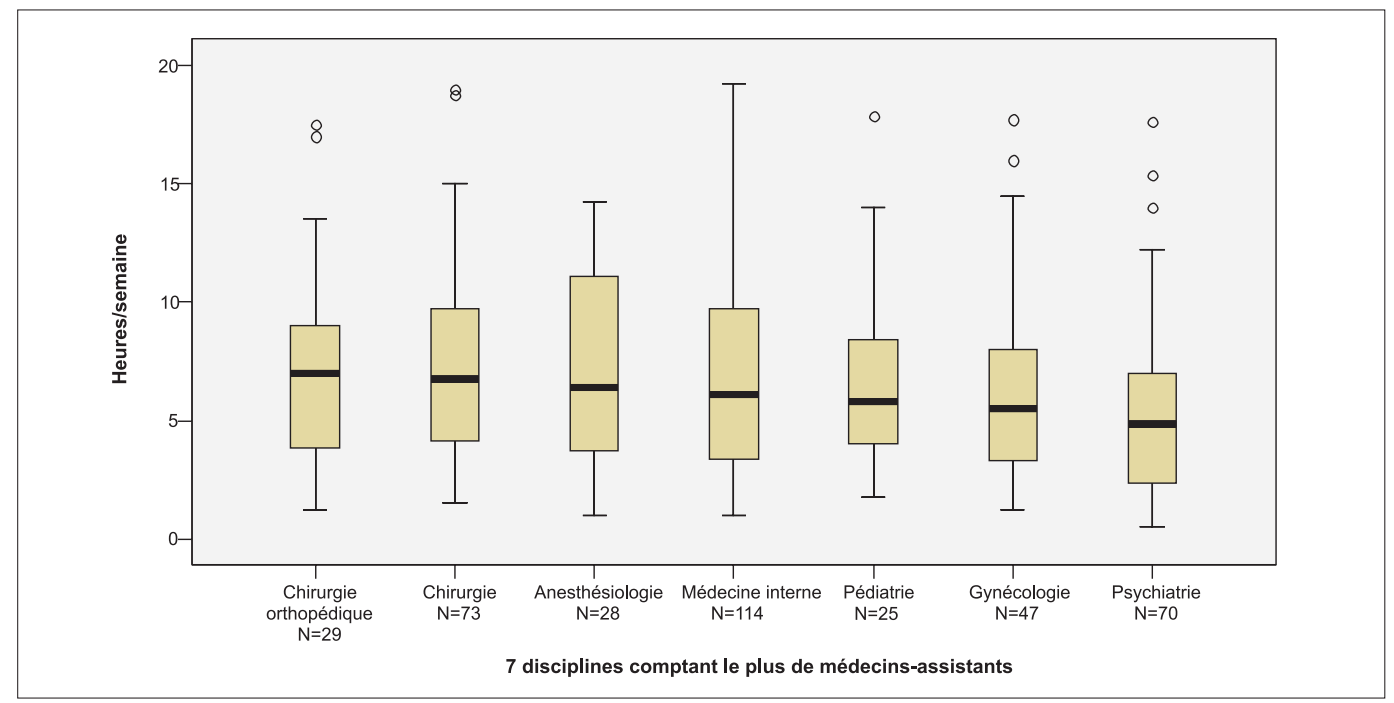

\section{Annexe}

\section{Questionnaire de l'enquête effectuée en 2006 auprès des responsables d'établissements}

Les responsables d'établissements de formation postgraduée ont répondu aux questions figurant ci-après.

\section{Question \\ 1. Quelle est votre fonction? \\ 2. Avez-vous reçu un rapport sur l'enquête 2005 contenant les résultats concernant votre établissement de formation postgraduée? \\ 3. Parmi les activités suivantes, lesquelles ont-elles été entreprises à la suite de ce rapport? (possibilité de nommer plusieurs domaines)}

4. Ce rapport m'a permis d'apprendre quelque chose de nouveau sur notre établissement de formation postgraduée

5. Notre établissement de formation postgraduée a été évalué correctement dans l'enquête

6. Les résultats de l'enquête $\mathrm{m}^{\prime}$ ont incité/e à réfléchir à notre formation postgraduée

7. Les résultats de l'évaluation 2005 ne sont pas pertinents sur le plan statistique en ce qui concerne notre établissement, car la participation des assistants était trop faible

8. Durant le temps de travail réglé par contrat, les médecins-assistants exécutent leurs tâches à mon entière satisfaction

9. La réglementation du temps de travail régie par la loi a des effets négatifs sur la formation postgraduée des médecins-assistants

10. La réglementation du temps de travail régie par la loi a des effets négatifs sur les soins donnés aux patients par les médecins-assistants

11. Arrive-t-il que vos médecins-assistants ne puissent pas respecter les temps de repos prescrits par la loi sur le travail?

12. Le nombre d'heures de travail fixé dans le contrat de travail (taux d'occupation à 100\% = au maximum 50 heures/semaine) est-il respecté chez les médecins-assistants?

13. Combien d'heures consacrez-vous en moyenne par mois à la formation postgraduée des médecins-assistants pour les activités suivantes?

\section{Réponses possibles \\ Médecin-chef; médecin adjoint; chef de clinique; autre \\ Oui; non}

Le rapport n’a pas encore été lu; le rapport a été lu; les résultats ont été discutés avec les chefs de clinique/médecins-adjoints; les résultats ont été discutés avec les médecins-assistants, des mesures concrètes ont été prises pour améliorer la qualité de la formation postgraduée; des lettres/critiques/ demandes ont été adressées à la FMH/à I'Institut de recherches sociales de I'Université de Zurich

$1 c^{\prime}$ est totalement inexact; $2 ; 3 ; 4 ; 5 ; 6 c^{\prime}$ est parfaitement exact

$1 c^{\prime}$ est totalement inexact; $2 ; 3 ; 4 ; 5 ; 6 c^{\prime}$ est parfaitement exact

1 c'est totalement inexact; $2 ; 3 ; 4 ; 5 ; 6$ c'est parfaitement exact

1 c'est totalement inexact; $2 ; 3 ; 4 ; 5 ; 6$ c'est parfaitement exact

$1 c^{\prime}$ est totalement inexact; $2 ; 3 ; 4 ; 5 ; 6 c^{\prime}$ est parfaitement exact

1 c'est totalement inexact; $2 ; 3 ; 4 ; 5 ; 6$ c'est parfaitement exact

1 c'est totalement inexact; $2 ; 3 ; 4 ; 5 ; 6$ c'est parfaitement exact

jamais; très rarement; parfois; régulièrement

Oui; non

Entretiens concernant la planification de carrière des médecins-assistants: h/mois; entretiens de feedback concernant la formation postgraduée des médecins-assistants: $h$ /mois; réunions de cadres pour l'évaluation des médecins-assistants: $\mathrm{h}$ /mois; préparation personnelle pour la formation postgraduée des médecins-assistants: $h$ /mois; exposés pour médecins-assistants: $h$ /mois cours pour médecins-assistants: $\mathrm{h} /$ mois; instructions et directives au chevet du patient (bedside teaching/assistanat en chirurgie): $\mathrm{h} / \mathrm{mois}$ 